\title{
Wireless Power Transfer System
}

\author{
Hiroyuki Arai
}

\begin{abstract}
This paper presents a survey of recent wireless power transfer systems. The issue of wireless power transfer is to achieve a highly efficient system with small positioning errors of the facilities setting. Several theories have been presented to obtain precise system design. This paper presents a summary of design theory for short range power transfer systems and detailed formulations based on a circuit model and an array of infinitesimal dipoles. In addition to these theories, this paper introduces a coil array scheme for improving the efficiency for off axis coils. In the microwave range, tightly coupled resonators provide a highly efficient power transfer system. This paper present sanoverlay resonator array consisting of half wavelength microstrip line resonators on the substrate with electromagnetically coupled parasitic elements placed above the bottom resonators. The tight couplings between the waveguide and the load resonator give strong power transmission and achieve a highly efficient system, and enables a contact-less power transfer railroad. Its basic theory and a demonstration of a toy vehicle operating with this system are presented. In the last topic of this paper, harmonic suppression from the rectenna is discussed with respect to acircular microstrip antenna with slits and stubs.
\end{abstract}

Key words: Wireless Power Transfer, Coil Array, Overlay Resonators, Rectenna, Harmonic suppression.

\section{Introduction}

Several solutions have been proposed for wireless power transfer using electromagnetic (EM) waves. For example, Felica utilizes electromagnetically inductive coils [1] over a very short distance. One of the hottest issues is non-radiative mid-range energy transfer [2]. This has weak tolerance for positioning and the coverage area is limited near the coil, however, it is a good candidate for high power transfer for fixed to fixed point applications. After its demonstration by the MIT group, many researchers carried out further studies and presented design methods.

This is sometimes referred toas "magnetic field resonance"; however, it is simply understood as the strong coupling between two resonators. Its transfer efficiencydepends heavily on the distance between coils and is around $90 \%$ at $1 \mathrm{~m}$ and $40 \%$ at $2 \mathrm{~m}$ [2]. Although substantial electric power transmission is expected to charge an electric vehicle, a standard of small power less than $5 \mathrm{~W}$ was provided by the consortium [3]. In order to charge mobile devices, the detection of the device position is a key issue. The movement of coupling coils and the coil array are major schemes for free positioning of the device to be charged. Several applications using these methods are already on the market as cell phone chargers.
A basic theory for coil coupling is given by circuit theory [2], [4], and is also explained by a filter theory [5] and others. This paper presents a simple circuit theory based one lectromagnetism to find the coupling efficiency between coils. In practical power transfer applications, the efficiency is a very important factor. To improve the transfer efficiency, an parameter optimization of the coil [6] and a coil array approach have been presented [7], [8]. This paper also introduces a coupling experiment by coil array.

If the size of the coupling coil is very small compared with the wavelength, then it is approximated by an infinitesimal magnetic current element. The reciprocity theorem indicates that a pair of infinitesimal current elements, such as short dipoles, is also used for wireless power transfer [9]. This short dipole array is simply analyzed by a pair of infinitesimal currents [10] and is formulated by spherical mode expansion [11]. This paper introduces an approximate theory and presents its coupling characteristics.

The above systems provide efficient power transfer between fixed points. Another request is power transfer to moving vehicles, such as automobiles and trains. This is the so-called wireless power transfer rail system. The power supply rail was developed to enhance the degree of freedom in the installation of lighting fixtures [12].

Manuscript received August 5, 2011 ; revised August 18, 2011. (ID No. 20110805-01J)

Department of Electrical and Computer Engineering, Yokohama National University, Yokohama, Japan.

Corresponding Author : Hiroyuki Arai (e-mail : arai@ynu.ac.jp) 
The rail consists of two long lines that provide the voltage source. The rail and the object to be charged have a contact point. Non-contact power transfer is desirable for safety and maintenance. A hybrid coupler approach [13] and overlay microstrip resonators [14] have been proposed for this application. This paper presents a power rail application and its principle for contactless power transfer.

Long range power transfer systems, such as solar power satellite systems, require highly efficient RF to DC conversion [15], [16]. A key technology to improve this efficiency is the development of a rectenna with high efficiency [17]. A filter embedded into a rectifier circuit will suppress harmonic radiation due to the non-linearity, while modified shaped patch antennas also suppress harmonic radiation. For the last topic of this paper, we examine the effects of harmonic suppression effects by the antenna shape and filters. The total conversion efficiency is demonstrated by measuring DC output voltage.

This paper first presents basic theories for a short range wireless power transfer system for fixed to fixedpoint applications. A simple circuit theory is introduced to design the coupling between coils. A coil array to improve coupling efficiency is also introduced. Another short range power transfer system, an approximated formula of electrically short dipole array, is demonstrated. For the power transfer to moving vehicles, a contactless power rail system and its principle are presented. A free access mat, our original power rail concept, is discussed in detail. The last section is devoted to a basic device in the power transfer system, namely a rectenna design to increase the RF to $\mathrm{DC}$ conversion efficiency. This paper concludes with a discussion of the issues of wireless power transfer systems.

\section{II . Theory of Short Range Power Transfer}

\section{2-1 Theory of Coil Coupling}

A simple model for coupled coils is presented in Fig. 1 , where matching capacitance $C_{o}$ is added to each feeding point to obtain a series resonance for the coil [4]. In the practical application, a feeding one-turn loop is used for input matching of the coil [2]; however, this is simplified by inserting the capacitance in this analysis model. An equivalent circuit model for Fig. 1 is presented in Fig. 2.

Assuming radiation loss only in this circuit, the radiation resistance of each coil is given by a formula for an electrically short normal mode helical antenna:

$$
R_{r}=\left(25.3 \frac{h}{\lambda}\right)^{2}
$$

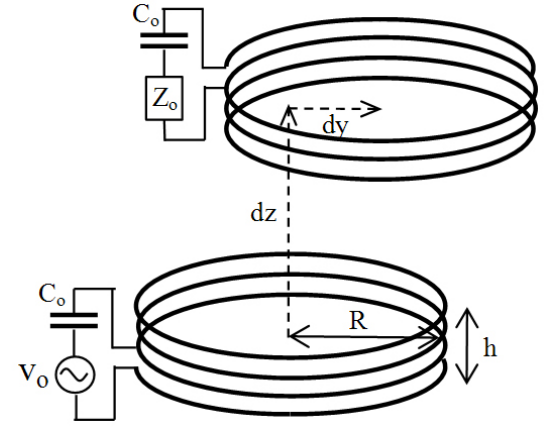

Fig. 1. Two coils for power transfer.

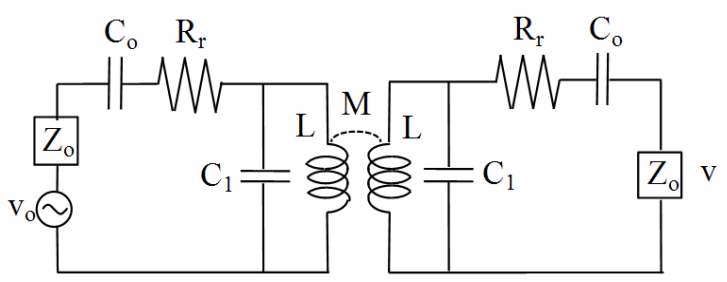

Fig. 2. Equivalent circuit for two coupled coils.

The self and mutual inductance of coupled coils are calculated by Neumann's formula as:

$$
L, M=\frac{\mu_{o}}{4 \pi} \oint \oint \frac{d \mathbf{s}_{1} \cdot d \mathbf{s}_{2}}{r}
$$

where $d s_{1}$ and $d s_{2}$ are the elements along each coil and $r$ is the distance between $d s_{1}$ and $d s_{2}$. A floating capacitance $C_{o}$ of each coil is given by approximating N-turn coils as thin conductor slices, as:

$$
C_{1}=(N-1)^{2} \frac{\varepsilon_{o} \pi R^{2}}{h}
$$

The coupling characteristics of two coils are calculated by these formulas, and are shown in Fig. 3 as a function of coil distance.

The coupling distance is enhanced by the increase in coil turn, so the design parameters should be adjusted to fix the power transfer distance. The frequency characteris-

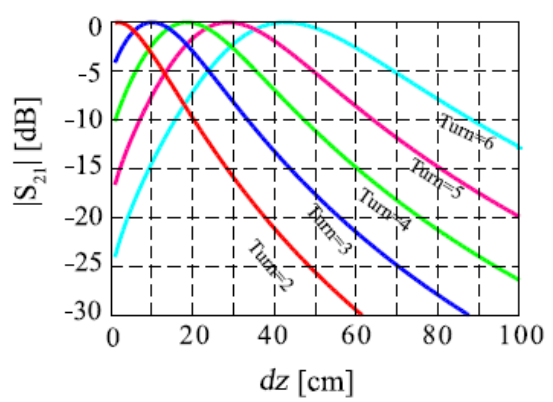

Fig. 3. Coupling between two coils, $d y=0, R=150, h=50$ $\mathrm{mm}, f=10 \mathrm{MHz}$ [4]. 


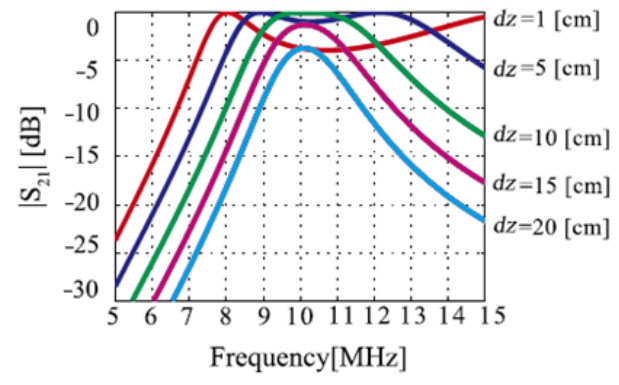

Fig. 4. Frequency characteristics of coupling between two coils, $d y=0, R=150, h=50 \mathrm{~mm} \mathrm{[4].}$

tics are shown in Fig. 4. Small coil spacing increases the mutual coupling and causes dual resonances. The coupling distance is seriously changed by the turn number and frequency, and we need to adjust the coil diameter and thickness when the operating frequency and coil distance are given in advance.

The power coupling characteristics of the two coils are calculated by this simple formula; however, the circuit losses in the real system are not precisely estimated.

In addition to the circuit loss, a misalignment of the two coil axes reduces the coupling efficiency. To overcome this difficulty, a transmission coil array was proposed [7], [8]. Fig. 5 shows the coil array system and Fig. 6 shows its experimental setup. Each coil is excited by a small loop for impedance matching.

A key feature of this array system is an excitation of transmission coils. To improve an off axis reception coil, two coils are excited in phase or out of phase. Fig. 7 shows the measured transfer efficiency for the orientation angle $\theta$ from 0 to $90^{\circ}$. The transfer efficiency of a single transmission coillocated at the center between coil \#1 and \#2 is also measured for comparison.

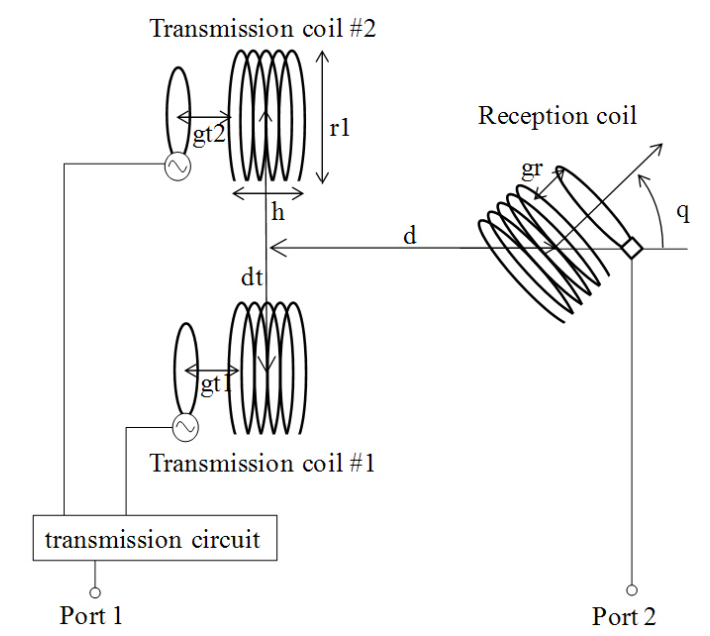

Fig. 5. Wireless power transfer system using a transmission coil array [8].

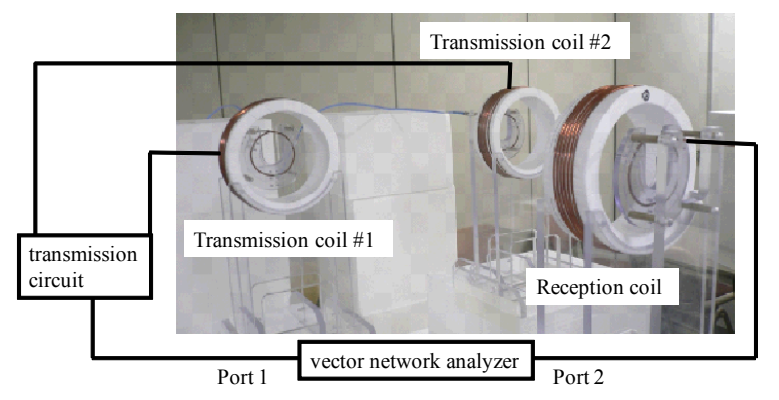

Fig. 6. Photo of measurement system [8].

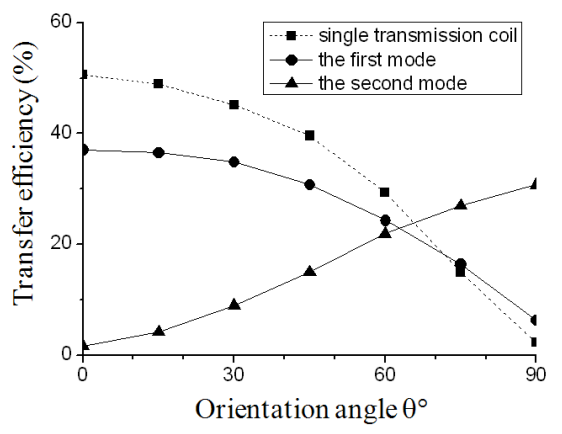

Fig. 7. Measured transfer efficiency, the first mode is in phase excitation and the second mode is out of phase excitation [8].

The normalized distance defined as $d n=d / r 1$ is 2.67 and $d t n=2.67$. The maximum transfer efficiency is obtained around $27 \mathrm{MH} \mathrm{z}$ in Fig. 7. In this system, the first mode is a suitable mode for the orientation angle $\theta<60^{\circ}$. For $\theta<60^{\circ}$, the second mode should be chosen to increase the efficiency. This coil array system has higher transmission efficiency for $\theta>70^{\circ}$.

\section{2-2 Theory of Short Dipole Array}

A pair of electrically short dipoles is another candidate for a short range power transfer system. This section presents an analysis of wireless power transmission by a pair of small dipoles, approximated as infinitesimal current filaments [10]. The analysis model is shown in Fig. 8, where the antenna array is approximated as a pair of infinitesimal current filaments.

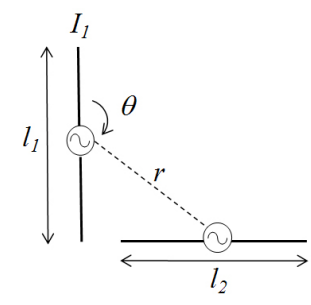

Fig. 8. Geometry of a pair of small dipoles. 


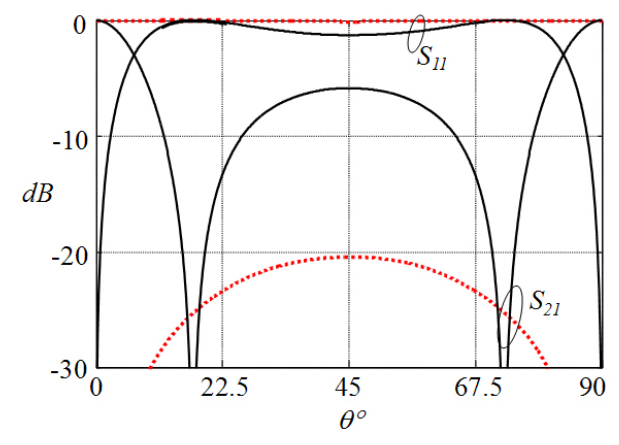

Fig. 9. $S$-parameters of dipole array, $r=0.0452 \lambda$ for solid lines, $r=0.0136 \lambda$ for lines $l_{1}=\lambda / 64, l_{2}=\lambda / 128, f=$ 13.6 MHz.

The input and mutual impedances of this dipole array are given as follows:

$$
Z_{i i}=20\left(k_{o} l_{i}\right)^{2}-\frac{240}{k_{o} l_{i}}\left(\ln \frac{l_{i}}{2 a}-1\right), \quad Z_{12}=-\frac{1}{I_{1} I_{2}} \int E_{12} d s
$$

where $i=1,2$ and a is the dipole wire radius. The $E_{12}$ is the induced electric field at the \#1 feed point by antenna $\# 2$, and is approximated by excited fields of infinitesimal current filaments. As the impedance matching condition, the imaginary part is ideally canceled to calculate the $2 \times 2 S$-parameter matrix. The $S$-parameter characteristics are shown in Fig. 9, where the angle $\theta$ is changed. When the antenna distance is small, the two antennas have strong coupling at $\theta=0,90^{\circ}$. A right angle array $\left(\theta=90^{\circ}\right)$ provides an interesting application; however, the input impedance matching in an actual circuit is left as a future problem.

\section{Flexible Free Access Mat}

This section presents a novel wireless power transfer system consisting of tightly coupled microstrip line resonators. Half wavelength microstrip line resonators are arrayed on the substrate and are electromagnetically coupled by a parasitic resonator placed above the bottom resonators, as shown in Fig. 8. The original structure is based on aribbon-wire interconnected with a DC cut [18]. These overlay resonators are used as a one-dimensional waveguide, providing the power to another microstrip resonator just above it. The tight coupling between the waveguide and the load resonator gives strong power transfer and results in a highly efficient system. The 1-D waveguide geometry facilitates the application of curved lines, which provides a contact-less power transfer railroad.

The configuration of the free access mat for the ISM band is shown in Fig. 10 (a) and (b). We use the infinite ground plane only for the simulation. Each port is

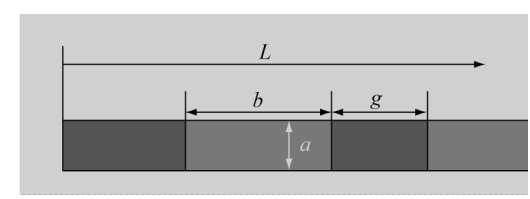

(a) Top view

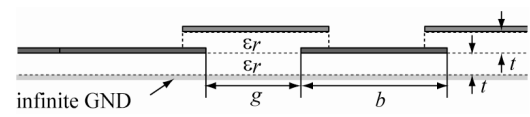

(b) Side view

Fig. 10. Geometry of overlay coupled microstrip resonators.

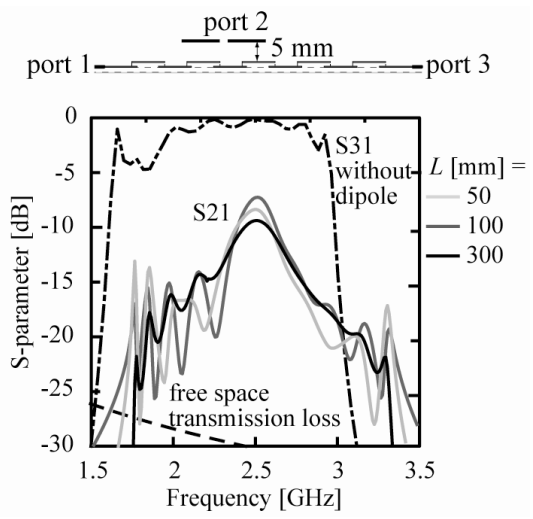

Fig. 11. $S$ parameters of $1-\mathrm{D}$ free access mat, $a=5, b=$ 46, $g=24.5, t=0.8 \mathrm{~mm}, \varepsilon_{r}=2$.

perfectly matched to the patch elements terminated by $50 \Omega$. The port 2 is a half wavelength dipole antenna that picks up the RF energy from this wave guide. Fig. 11 shows the simulated transmission loss $\left(S_{31}\right)$ along the one-dimensional free access mat and the coupled power level at the dipole antenna. The transmission loss is smaller than $1 \mathrm{~dB}$ over awide frequency range and the coupled power level is around $-10 \mathrm{~dB}$ due to re-radiation by the dipole antenna.

A model vehicle run by this waveguide is demonstrated with a rectenna embedded into a square microstrip resonator as a load. The receiving part consists of the antenna, filter, rectifier, and load. GaAs FET and HEMET, as well as Schottky diodes, are applied to the rectifiers. HSMS-282 series are good for the detector applications, with input power levels greater than -20 $\mathrm{dBm}$ at frequencies below $4.0 \mathrm{GHz}$ [19].

A simple LPF (Low Pass Filter) is inserted between the receiving antenna and the rectifying circuit to suppress the harmonics re-radiation from the rectifier. The coupled power level is increased to $-6 \mathrm{~dB}$ for the patch antenna by the suppression of re-radiation.

We demonstrated wireless power transfer by our proposed system by building three types of waveguides, as shown in Fig. 12, where two different length straight 


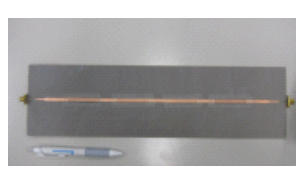

(a) $L=38 \mathrm{~cm}$

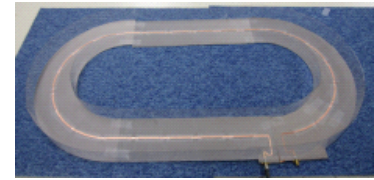

(b) $L=228 \mathrm{~cm}$ (along line)

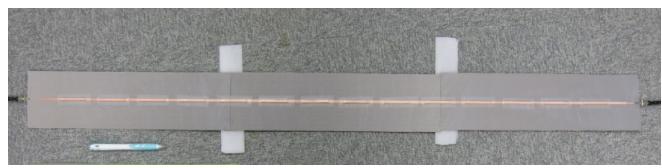

(c) $L=109 \mathrm{~cm}$

Fig. 12. Photos of three waveguide.

guides (a), (c) and a race track guide (b) are examined. Measured insertion loss $\left(S_{31}\right)$ of the three guides are (a) $-1 \mathrm{~dB}$, (b) $-7 \mathrm{~dB}$ and (c) $-4 \mathrm{~dB}$ due to the unwanted radiation from the guide. The long guide has a radiation loss of $3 \mathrm{~dB}$ larger than the short one. The insertion loss of the race track depends on the guide length and is not increased by the existence of four corners. A toy vehicle run is successfully demonstrated by this waveguide.

Not all of the input power from the RF source is supplied to the vehicle. The rest of the power at the waveguide end is terminated to a dummy load in the measurement. To increase the total efficiency of the system, a power recollecting system is introduced [20]. A simple one is provided by connecting the guide end to the rectifier to collect the rest of the energy as DC. Another system is a positive feedback system that returnsthe rest of the power after adjusting its phase. The phase of $S_{21}$ fluctuates between $110^{\circ}$ and $160^{\circ}$ depending on the position of the toy vehicle. A real-time phase control is not easy to introduce into this system, and we fix the feedback phase as an average value of the above. The phase shifter consists of a microstrip delay line. A few $\mathrm{dB}$ amplitude deviations are also observed; however, we do not use any amplitude feedback control for the simple system. To escape from an oscillation of the circuit by continuous feedback, a switching circuit is introduced into this system. The switching circuit automatically checks the input voltage to the guide and suppresses the oscillation. The circuit diagram is shown in Fig. 13. The power feedback gain of this collecting circuit is around 2 to $3 \mathrm{~dB}$, which is effective for enhancing the total efficiency of the system.

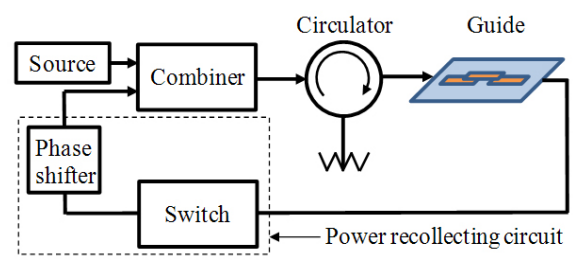

Fig. 13. Power recollecting system.

\section{Conversion Efficiency of the Rectenna}

In wireless power transfer systems, the receiving antenna with a rectifier, called the rectenna, has been investigated as a way to attain high conversion efficiency by suppressing re-radiation of harmonics excited in the diodes of rectifying circuits. For high conversion efficiency, circular microstrip patch antennas (CMA) have been proposed with slits [21], [22] or stub loadings. However, the combination of slits and stubs has not yet been examined for suppression of re-radiation of harmonics. Very few papers have evaluated the effect of suppressing harmonics by measuring antenna efficiency. To confirm whether the efficiency is improved by antenna differences, we measured RF-DC conversion efficiency.

High power wireless transfer, requires the development of highly efficient rectennas. A typical rectenna consists of a receiving antenna, a LPF (Low Pass Filter) for the fundamental mode, a diode, a DC pass filter, and a resistive load. The most important issue in the rectenna design is obtaining high efficiency. Approaches like broadband antennas [23], large antenna arrays [24], and circular polarized antennas [25] have been investigated in the past several years to achieve this goal. Another approach includes the suppression of harmonics generated by the nonlinear characteristics of the diode. This causes alow conversion efficiency; therefore, the rectenna requires an LPF between the antenna and the diode, and a DC pass filter on the diode output side.

One merit of the circular microstrip antenna (CMA) is that higher mode resonance frequencies do not coincide with harmonics of the diode. However, the resonance band edges overlapping with harmonic frequencies reduce the conversion efficiency. Suppression of the second and third mode resonances of CMA is very effective for improving the conversion efficiency. We examined four types of CMA for the rectenna in order to suppress harmonics in the rectifying circuit. The geometry of the CMA is shown in Fig. 14. We also demonstrated an improved efficiency by applying stubs and slits to the CMA. The techniques for suppression of the resonances at the second and third higher order modes are given by inserting slits in the CMA by controlling the current flows on the top patch [22]. The ideal shape of the slit is parallel to the dominant current flow and is perpendicular to higher order mode currents. The current distributions on the CMA are complex and the harmonics of re-radiation are not completely suppressed by this technique. Another harmonics suppression method is to add open stubs for the feeding microstripline [24]. The length of the open stub is a quarter guide wavelength in higher order resonant modes. Two or more stubs are required for complete suppression of the second and third modes. 


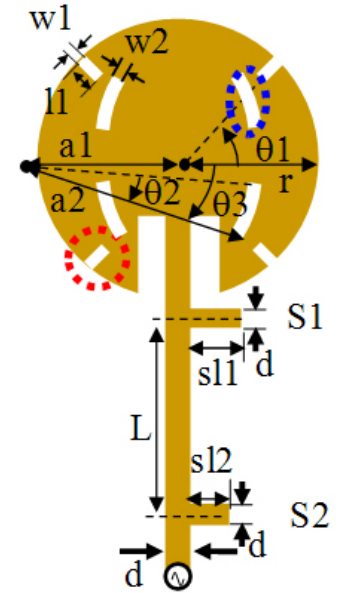

Fig. 14. Geometry of circular patch antenna with stubs and slits, $r=22.5, d=0.5 \mathrm{~mm}$.

We compare the effects of suppressing harmonics in the $S_{11}$ characteristics. Fig. 15 summarizes the $S_{11}$ of four types of CMA designed to have a resonance at 2.45 $\mathrm{GHz}$. The dielectric substrate of the relative constant $\varepsilon_{r}$ is 2.6 with the thickness of $1.6 \mathrm{~mm}$. Fig. 15(a) shows the $S_{11}$ of the original CMA structure and three higher order mode resonances that appear at less than $7 \mathrm{GHz}$. First, we add an open stub S1 with the length of $\lambda_{g} / 4$ at $6.7 \mathrm{GHz}$, where $\lambda_{g}$ is the guide wavelength. The deep resonance at $6.7 \mathrm{GHz}$ is reduced, while another resonance at $6.1 \mathrm{GHz}$ is excited. To eliminate this, we add another stub, $S_{2}$, which improves the $S_{11}$ at the second and third harmonics, as shown in Fig. 15(b). Next, we examine the slits as another harmonic suppression technique. To reduce current flows of the CMA at the second mode frequency at $4.05 \mathrm{GHz}$, the slits are cut as shown in Fig. 11, enclosed with a red dotted line. The resonance at $4.05 \mathrm{GHz}$ is well suppressed, while the resonance at $6.7 \mathrm{GHz}$ is not changed. To suppress the currents at $6.7 \mathrm{GHz}$, we add arch shaped slits, as shown in Fig. 14, enclosed with a blue dotted line. Fig. 15 (c) shows its $S_{11}$ characteristics. It suppresses the resonances at higher order mode frequencies, where the diameter of CMA and slit parameters are adjusted to suppress the resonance at $4.05 \mathrm{GHz}$. We then examine the CMA with slits and stubs. As shown in Fig. 15(c), the CMA with two kinds of slits has resonances at $3.6 \mathrm{GHz}, 4.45$ $\mathrm{GHz}$ and $6.4 \mathrm{GHz}$. We add stubs to CMA with slits, just like above method. This optimization reduces these resonant frequencies, as shown in Fig. 15(d). The CMA with slits and stubs is the best choice to suppress the higher order mode resonances for the CMA. As a result, the $S_{11}$ of the second and third harmonic frequencies is suppressed to $-0.26 \mathrm{~dB}$ at $4.9 \mathrm{GHz}$ and $-0.17 \mathrm{~dB}$ at $7.35 \mathrm{GHz}$.

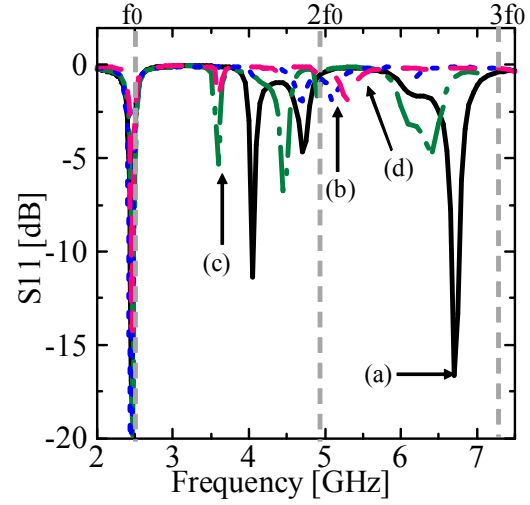

Fig. 15. Geometry of circular patch antenna with stubs and slits, (a) Without slits and stubs, (b) With stubs and without slits $\left(s l_{1}=6.9, s l_{2}=7.6, L=26\right.$, $\left.w_{1}=w_{2}=l_{1}=0\right)$, (c) With slits and without stubs ( $\theta$ ${ }_{1}=45^{\circ}, \theta_{2}=15^{\circ}, \theta_{3}=20^{\circ}, w_{1}=w_{2}=0.5, l_{1}=4.5, s l_{1}=$ $\left.s l_{2}=0, a_{1}=35, a_{2}=47\right)$, and (d) With slits and stubs $\left(\theta_{1}=45^{\circ}, \quad \theta_{2}=15^{\circ}, \quad \theta_{3}=20^{\circ}, \quad w_{1}=w_{2}=0.5, \quad l_{1}=4.5\right.$, $\left.s l_{1}=10.5, s l_{2}=7.4, L=22, a_{1}=35, a_{2}=47\right)$. Units are $\mathrm{mm}$.

To measure the power conversion efficiency, a rectifying circuit is designed, as shown in Fig. 16. The rectifying circuit is connected to the antenna, and we measure RF-DC conversion efficiency. The above design procedure suppresses the second and third harmonics of CMA, then the LPF between the antenna and diode is eliminated. The RF-DC conversion efficiency is defined as $\eta=V_{\mathrm{o}}^{2} /\left(R_{L} P_{R F}\right)$, where $V_{o}$ is the output voltage at the load, $R_{L}$ is the variable resistor value, and $P_{R F}$ is the input power as shown in Fig. 13. We join this rectifying circuit and 4 types of antennas, where a square microstrip antenna is measured as a reference. Fig. 17 shows conversion efficiency for the input power level, where a simulated ideal curve is calculated by approximating the antenna as an ideal LPF. In this calculation, the value of $R_{L}=1,500 \Omega$. The maximum conversion efficiencies of each curve are $55.8 \%$ (ideal curve), $43.6 \%$ (square MSA as a reference), $47.5 \%$ (original CMA), $51.8 \%$ (CMA with stubs) and $53.0 \%$ (CMA with slits and stubs), respectively. This result shows that the efficiency of the CMA with slits and stubs is higher than that of the other antennas.

In this section, we showed that CMA suppresses reradiation of harmonics excited by the diode in a rectifying circuit. We examined the input characteristic of the CMA by slits, stubs, and their combinations and showed that the CMA with slits and stubs was the most effective in suppressing harmonics. We also measured RFDC conversion efficiency, and confirmed that the conversion efficiency is improved by applying stubs and slits to the CMA. 


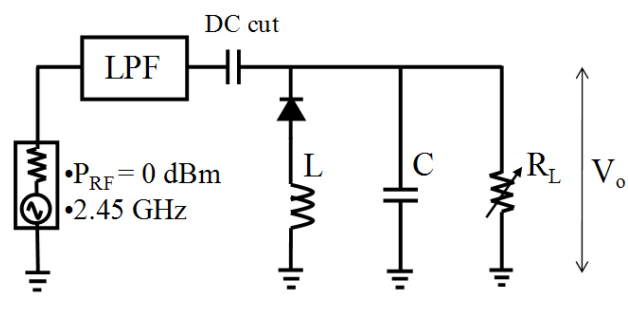

Fig. 16. Rectenna circuit.

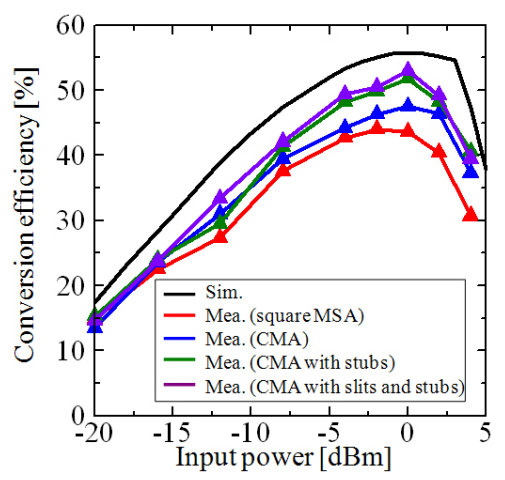

Fig. 17. RF-DC conversion efficiency.

\section{Conclusion}

This paper presented three topics in the research fields of wireless power transfer. A simple circuit theory was introduced to explain short range power transfer by the resonator coupling. The theory, based on electromagnetics, is easy to understand for electrical engineers, and provides a simple design tool for the coupling between two coils. To overcome misalignment of the coupling coils, a two-element array coil is a good candidate for practical applications. A further improvement in coupling efficiency is expected to result in a highly efficient power transfer system. Short range power coupling is also given by a pair of electrically small dipole arrays. A theory based on infinitesimal current filaments was presented and its flexible coupling scheme was demonstrated. Its experimental verification is fully expected.

The power rail concept without physical contact is a very attractive technology for delivery of power to moving vehicles. This paper demonstrated a new power rail concept that uses coupled overlay microstrip resonators and that provided small insertion loss and tight coupling to the load rectenna. A toy vehicle was successfully powered by this rail. The decrease in operating frequency is an important issue for this power rail application and a power recollecting system in the RF range is a major challenge forachieving high power wireless transfer.

The last topic of this paper was the rectenna efficiency in the microwave range as a means of long range power transmission. The RF to DC conversion effi- ciency is mainly determined by the performance of the rectifier diode. We have already designed a highly efficient rectenna that operates in a high power handling region of more than a few watts [27], and it reaches to around $90 \%$ at $2.45 \mathrm{GHz}$. However, we have still have requests to improve the conversion efficiency of the rectenna in the low power microwave region, as discussed in this paper. Optimal design for suppression of the harmonic radiation from rectifiers is also an important research topic in rectenna design.

Wireless power transfer has become a hot research topic recently for electromagnetic wave researchers. Through their contributions, Tesla's lost dream could come true, step by step, in the near future. After decades of "All information is wireless," a new age of wireless power transfer is on its way.

The author would like to thank Prof. N. Kikuma, Nagoya Institute of Technology and Dr. Oodachi, Toshiba Corporation to permit to use their figures.

\section{References}

[1] www.sony.net

[2] A. Kurs, A. Karalis, R. Moffatt, J. D. Joannopoulos, P. Fisher, and M. Solja I, "Wireless power transfer via strongly coupledmagnetic resonances," Science, vol. 317, no. 5834, p. 83, Jul. 2007.

[3] www.wirelesspowerconsortium.com

[4] Y. Hiraiwa, N. Kikuma, H. Hirayama, and K. Sakakibara, "A consideration on transmission efficiency characteristics of wireless power transmission using proximity coils," IEICE Technical Report, AP 200986, Sep. 2009.

[5] I. Awai, T. Ishida, "Design of resonator-coupledwireless power transfer system by equivalent circuit," Journal of The Korean Institute of Electromagnetic Engineering and Science, vol. 10, no. 4, pp. 237-243, Dec. 2010.

[6] T. Miyamoto, S. Komiyama, H. Mita, and K. Fujimaki, "Wireless power transfer system with a simple receiver coil," IEEE MTT-S IMWS on Innovative Wireless Power Transmission: Technologies, System, and Applications, IWPT6-5, May 2011.

[7] N. Oodachi, H. Kudo, K. Ogawa, H. Shoki, S. Obayashi, and T. Morooka, "Efficiency improvement of wireless power transfer via magneticresonance using the third coil," ISAP 2010, paper ID 52, Macao, China, Nov. 2010.

[8] N. Oodachi, K. Ogawa, H. Kudo, H. Shoki, S. Obayashi, and T. Morooka, "Efficiency improvement of 
wireless power transfer via magnetic resonance using transmission coilarray," 2011 IEEEAPS, Jul. 2011.

[9] T. Maruchi, N. Inagaki, and K. Fujii, IEICE Technical Report, AP2009-84, Sep. 2009.

[10] Hiroyuki Arai, "A remark on wireless transmission by a pair of small dipoles," 2010 Autumn Microwave \& Radio Wave Conference, Busan, Korea, Oct. 2010.

[11] J. Lee, S. Nam, "Fundamental aspect of near-field coupling small antennas for wireless power transfer," IEEE Transaction on Antennas and Propagation, AP-58, 11, pp. 3442-3449, Nov. 2010.

[12] http://techon.nikkeibp.co.jp/article/NEWS/20080804/ 155967

[13] I. Awai, K. Hori, S. Yakuno, and K. Namikoshi, "Movable wireless power transmission by use of directional coupler," IEICE Technical Report, MW 2009-147, Dec. 2009.

[14] H. Arai, K. Eom, "Architecture and application of sheet-like waveguide," IEICE Technical Report, AP2007-50, Jul. 2007.

[15] P. E. Glaser, "Power from the sun: Its future," Science, vol. 162, pp. 867-886, 1968.

[16] DOE/NASA, "Program assessment report statement of finding - Satellite power systems, concept development and evaluation program," DOE/ER-0085, 1980.

[17] T. Ito, Y. Fujino, and M. Fujita, "Fundamental experiment of a rectenna array for microwavepower reception," IEICE Trans. Commun., vol. 44, no. 3, pp. 105-111, Sep. 1998.

[18] H. Izumi, H. Arai, and T. Itoh, "Ribbon-wire interconnect using parasitic element," Trans. IEICE Japan, vol. E-82-C, no. 4, pp. 662-664, Apr. 1999.

[19] J. O. McSpadden, K. Chang, "Suppression of rec- tenna harmonic radiation by a frequency selective surface," 1992 IEEE/APS/URSI/NEM Joint Symposia, Chicago, 1992.

[20] H. Arai, N. Yoneyama, "Wireless power transmission system by tightly coupled microstrip line overlay resonators," IEEE MTT-S IMWS on Innovative Wireless Power Transmission: Technologies, System, and Applications, IWPT4-1, May 2011.

[21] K. Itoh, Y. Akiba, and Y Ogawa, "On rectennas using circular microstrip patch antennas," IEICE Tech. Report, AP1982-98, Nov. 1982 (in Japanese).

[22] Y. Yamada, Syahrial, M. Omiya, and K. Itoh, "Characteristics of a circular microstrip patch antenna with slits," IEICE Tech. Report, AP1997-65, Jul. 1997 (in Japanese).

[23] J. A. Hagerty, Z. Popovic, "An experimental and theoretical characterization of a broadband arbitrarily-polarized rectenna array," in Proc. IEEE MTTS Int. Microwave Symp. Dig., Phoenix, AZ, vol. 3, pp. 1855-1858, Jun. 2001.

[24] N. Shinohara, H. Matsumoto, "Experimental study of large rectenna array for microwave energy transmission," IEEE Trans. Microwave Theory Tech., vol. 46, pp. 261-268, Mar. 1998.

[25] B. Strassner, K. Chang, "5.8-GHz circularly polarized dual-rhombic-loop traveling-wave rectifying antenna for low power-density wireless power transmission applications," IEEE Trans. Microwave Theory Tech., vol. 51, pp. 1548-1553, May 2003.

[26] F. L. Hsiao, T. W Chiou, and K. L. Wong, "Harmonic control of a square microstrip antenna operated at $1.8 \mathrm{GHz}$ band," 2001 Asia-Pacific Microwave Conference, pp. 1052-1055, Dec. 2001.

[27] www.den-gyo.com 
Hiroyuki Arai

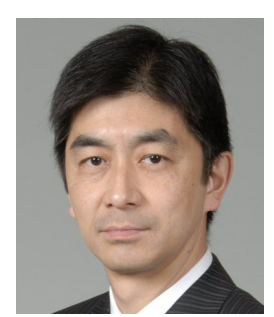

received the B.E. degree in Electrical and Electronic Engineering, M.E. and D.E. in Physical Electronics from Tokyo Institute of Technology in 1982, 1984 and 1987, respectively. After a research associate in Tokyo Institute of Technology, he joined to Yokohama National University as a lecturer in 1989. Now he is a professor in Division of Electrical and Computer Engineering, Yokohama National University. He was a visiting scholar at University of California, Los Angeles in 1997 and was visiting professor at Yonsei University, Seoul in 2005. He investigated microwave passive components for high power handling applications such as RF plasma heating in large Tokamaks. He developed a flat diversity antenna for mobile telephone terminal, a polarization diversity base station antenna for Japanese PDC systems, small base station antennas of in-building micro cellular system and DOA estimation for cellular system. His current research interest includes MIMO antennas, wireless power transmission, energy harvesting in EM waves and EMC/EMI antennas. He is the author of five edited books, three research book chapters, over 130 journal papers and 1,000 conference papers. He was awarded 5 US and more than 50 Japanese patents. 